\title{
EVALUACIÓN DE LAS PRÁCTICAS DE CUIDADO MATERNO INFANTIL EN ÁREAS CON POBREZA EXTREMA DEL PERÚ, 2012
}

\author{
José Enrique Velásquez Hurtado1,2,a,b, Lucía Solís Alcedo1,a,b, Walter Eduardo Vigo Valdez ${ }^{1, a, c}$, \\ Ángel Martín Rosas Aguirre ${ }^{2, a, b}$, Paulina Giusti Hundskopf ${ }^{1, a, b}$, \\ Paul Alfaro Fernandez $z^{1, a, d, e}$, Henry Cabrera Arredondo ${ }^{1, a, b}$
}

\begin{abstract}
RESUMEN
Objetivos. Evaluar las prácticas de cuidado materno-infantil en áreas con pobreza extrema del Perú. Materiales y métodos. Estudio transversal realizado entre agosto y septiembre de 2012, con muestreo probabilístico, trietápico y estratificado por departamento. Se seleccionaron 540 hogares que tenían al menos un niño menor de 36 meses (475 hogares) y/o mujer gestante (80 hogares) en áreas rurales de Cajamarca, Amazonas, Huánuco, Ayacucho, Huancavelica, Apurímac, Cusco, Puno y Ucayali. Resultados. Con relación al último embarazo, 69,0\% de la madres refirió haber tenido su primer control prenatal en el primer trimestre; $65,3 \%$ haber cumplido más de seis controles a lo largo del embarazo; $81,1 \%$ haber dado a luz en un establecimiento de salud, y solo $31,0 \%$ haber elegido un método de planificación familiar dentro de los 42 días posparto. Con relación al último niño menor de 3 años, $64,1 \%$ tuvo contacto precoz madre-niño, y $62,8 \%$ inició la lactancia materna dentro de la hora de nacido. Además, $89,6 \%$ de los niños menores de 6 meses recibía lactancia materna exclusiva, y 89,1\% de los niños de 6 a 8 meses ya tenía alimentación complementaria. Fiebre, diarrea, vómitos y dificultad respiratoria fueron las señales de peligro más referidas para la búsqueda de atención en niños. Conclusiones. La evaluación ha permitido establecer una línea de base sobre la cual se diseñará e implementará una estrategia que posibilite una mejora en las prácticas clave de cuidado materno-infantil en la población objetivo del Programa de Apoyo a la Reforma del Sector Salud.
\end{abstract}

Palabras clave: Cuidado infantil; Salud materno-infantil; Prevención y control; Evaluación en salud (fuente: DeCS BIREME).

\section{EVALUATING MATERNAL CHILD CARE PRACTICES IN EXTREME POVERTY AREAS IN PERU, 2012}

\begin{abstract}
Objectives. To evaluate maternal and child care practices in areas with extreme poverty in Peru. Materials and methods. Cross-sectional study conducted between August and September 2012; with probabilistic, three-stage stratification by "department" (geographic region) sampling. 540 households were selected that had at least one child younger than 36 months (475 households) and/or a pregnant women ( 80 households), in rural areas of Cajamarca, Amazonas, Huanuco, Ayacucho, Huancavelica, Apurimac, Cusco, Puno and Ucayali. Results. Regarding the last pregnancy, $69.0 \%$ of the mothers reported having had their first prenatal care in the first trimester; $65.3 \%$ reported having completed more than six check-ups throughout the pregnancy; $81.1 \%$ reported having given birth in a health facility, and only $31.0 \%$ chose a method of family planning within 42 days postpartum. With regard to the last child under 3 years old, $64.1 \%$ had early mother-infant contact, and $62.8 \%$ initiated breastfeeding within one hour of birth. In addition, $89.6 \%$ of children under 6 months old exclusively received breastfeeding and $89.1 \%$ of children aged $6-8$ months old already had received complementary feeding. Fever, diarrhea, vomiting and breathing difficulty were the most mentioned warning signs for seeking care for children. Conclusions. Through this study, a baseline has been established on which a strategy can be designed and implemented to improve best practices for maternal and child care as part of the "Programa de Apoyo" within the Health Sector Reform.
\end{abstract}

Key words: Child care; Maternal and child health; Prevention and control; Health evaluation (source: MeSH NLM).

\footnotetext{
Programa de Apoyo a la Reforma del Sector Salud (PARSALUD II). Lima, Perú.

Universidad Peruana Cayetano Heredia. Lima, Perú.

Médico; ${ }^{\mathrm{b}}$ magíster en salud pública; ${ }^{\mathrm{c}}$ magíster en gerencia de proyectos y programas sociales; ${ }^{\mathrm{d}}$ magíster en administración en salud; ${ }^{\mathrm{e}}$ doctor en Medicina Recibido: 13-09-13 Aprobado: 12-03-14
}

Citar como: Velásquez Hurtado JE, Solís Alcedo L, Vigo Valdez WE, Rosas Aguirre AM, Giusti Hundskopf P, Alfaro Fernandez P, et al. Evaluación de las prácticas de cuidado materno infantil en áreas con pobreza extrema del Perú, 2012. Rev Peru Med Exp Salud Publica. 2014;31(2):243-53. 


\section{INTRODUCCIÓN}

A pesar del crecimiento económico en los últimos años, el Perú continúa con altos niveles de pobreza y una amplia brecha urbano-rural. En el 2012 se estimó que el $25,8 \%$ de la población vivía en situación de pobreza y $6,0 \%$ en extrema pobreza; sin embargo, mientras que la incidencia de pobreza en áreas urbanas es de 16,6\%, en áreas rurales se incrementa hasta un $53,0 \%{ }^{(1)}$. Si bien en el ámbito sanitario ha habido progresos, los valores de mortalidad materna e infantil y la desnutrición crónica en niños menores de cinco años aún están lejos de niveles adecuados, sobre todo en zonas pobres.

En el 2011 ocurrieron 92,7 muertes maternas por cada cien mil nacidos vivos; la mayoría de muertes fueron en mujeres de 30 o más años, en el tercer trimestre de embarazo y en las primeras $24 \mathrm{~h}$ del puerperio (2). Vinculado a la mortalidad materna, la mortalidad de los recién nacidos en el área rural (22 por mil nacidos vivos) es alta en comparación con sus pares urbanos (14 por mil nacidos vivos) ${ }^{(3)}$. Así mismo, aproximadamente uno de cada cinco niños menores de cinco años (18,1\%) presenta desnutrición crónica, prevalencia que es mayor en zonas rurales $(31,9 \%)$ donde se instala antes de los 24 meses de edad, por la elevada carga de morbilidad de infecciones agudas ${ }^{(4)}$.

El Programa de Apoyo a la Reforma del Sector Salud (PARSALUD) es un programa de inversión pública a cargo del Ministerio de Salud (MINSA), financiado con recursos del tesoro público y endeudamiento externo, provenientes de los Bancos Mundial e Interamericano de Desarrollo ${ }^{(5)}$. Actualmente los objetivos de la segunda fase del PARSALUD (PARSALUD II) buscan la reducción de la mortalidad materna, neonatal y la desnutrición crónica infantil en zonas rurales de los nueve departamentos más pobres del país. Sus actividades se agrupan en tres procesos: a) la promoción de prácticas adecuadas y recursos de cuidados familiares y comunitarios a mujeres (gestante, parturienta y madre en período de lactancia) y a niños menores de tres años; b) el mejoramiento de la capacidad resolutiva de las redes de servicios de salud, y c) el fortalecimiento de la capacidad de gobierno para el funcionamiento del sistema de salud. Para el logro del primer proceso, el PARSALUD ha previsto la incorporación de una estrategia de educación y comunicación para la promoción de prácticas clave y entornos saludables (ECPPCES) que contribuya con el incremento del uso de los servicios de salud materno-infantiles y la disminución de la desnutrición crónica.

El presente artículo presenta los resultados del estudio de línea de base para la estrategia ECPPCES, el cual tuvo como objetivo la evaluación de prácticas clave de cuidado materno-infantil; información que será utilizada para el diseño y posterior monitoreo y evaluación de la estrategia.

\section{MATERIALES Y MÉTODOS}

\section{DISEÑO DEL ESTUDIO}

Estudio transversal basado en encuestas a hogares realizado entre agosto y septiembre de 2012 en el ámbito de intervención del PARSALUD: zonas rurales de 202 distritos (316 451 hogares según censo nacional) ${ }^{(6)}$ de los nueve departamentos más pobres del país: Cajamarca, Amazonas, Huánuco, Ayacucho, Huancavelica, Apurímac, Cusco, Puno y Ucayali. Dichos departamentos concentran: el $53,9 \%$ de los menores de cinco años de las zonas rurales ${ }^{(6)}$; el $61,8 \%$ de la desnutrición crónica en menores de cinco años (3); el $54,9 \%$ de las gestantes de las zonas rurales ${ }^{(3)}$, y el $36,7 \%$ de las muertes maternas del país ${ }^{(7)}$.

\section{POBLACIÓN Y MUESTRA}

Todos los hogares del ámbito de intervención del PARSALUD tenían, al menos, un niño menor de 36 meses y/o mujer gestante. La muestra para la encuesta a hogares fue probabilística, trietápica, estratificada por departamento, con representatividad en el ámbito de intervención de PARSALUD. El tamaño muestral fue de 540 hogares con niños menores de 3 años y/o gestantes, considerando una prevalencia general de 0,5 , con un margen de error de $5 \%$, un intervalo de confianza de $95 \%$, un efecto de diseño de 1,2 y una tasa de no respuesta de $10 \%$.

En la primera etapa se seleccionaron 27 distritos del total de 202 distritos en los departamentos priorizados, mediante un muestreo aleatorio, proporcional a la cantidad de hogares por distrito. En la segunda etapa se seleccionaron aleatoriamente 27 centro poblados (uno por distrito), tomando en cuenta la cantidad de hogares por centro poblado. Previo a la tercera etapa, se realizó un censo de hogares en los centros poblados seleccionados, identificándose los hogares con niños menores de 3 años y/o con mujeres gestantes. La selección final de dichos hogares para la encuesta fue aleatoria hasta completar el tamaño muestral requerido por departamento.

\section{RECOLECCIÓN DE DATOS}

Encuestadores, previamente capacitados, visitaron los hogares seleccionados. Se entrevistaron a todas las madres de los niños menores de 36 meses y/o a las mujeres que se encontraban gestando al momento de la encuesta. En caso de ausencia de personas en un hogar en el primer intento de visita, se procedió a revisitar el mismo hogar al día siguiente. Para la encuesta de hogares se usó un cuestionario semiestructurado validado por juicio de expertos y en campo. El cuestionario estuvo compuesto por preguntas cerradas 
y abiertas orientadas establecer el estado actual de las prácticas claves de cuidado que el Ministerio de Salud del Perú recomienda durante en el embarazo, puerperio e infancia ${ }^{(8)}$; además de una sección para el registro de datos de las tarjetas de vacunación.

\section{ANÁLISIS ESTADÍSTICO}

Los datos registrados en el cuestionario fueron ingresados a una hoja de cálculo en Excel, en dos digitaciones independientes. Los hogares fueron clasificados según su nivel de pobreza mediante el método de necesidades básicas insatisfechas (NBI) ${ }^{(9)}$. En total se calcularon 16 indicadores de prácticas clave de cuidado (Tabla 1): tres durante el embarazo; dos durante el puerperio; dos para el recién nacido; siete para niños de 1 a 36 meses; uno relacionado al lavado de manos, y uno sobre el consumo de agua segura.

El procesamiento y posterior análisis fueron realizados con el paquete estadístico SPSS 15.0, siguiendo los procedimientos de estimación para muestreos por

Tabla 1. Indicadores de práctica de cuidado materno-infantil

\begin{tabular}{|c|c|}
\hline Indicador & Definición \\
\hline \multicolumn{2}{|l|}{ Cuidado durante el embarazo } \\
\hline $\begin{array}{l}\text { 1. Control prenatal en el primer } \\
\text { trimestre }\end{array}$ & $\begin{array}{l}\text { Número de madres que durante su última gestación tuvieron su primer control prenatal en el } \\
\text { primer trimestre/ número de madres encuestadas } \times 100 \text {. }\end{array}$ \\
\hline $\begin{array}{l}\text { Reconoce señales de peligro } \\
\text { 2. y busca ayuda en un estable- } \\
\text { cimiento de salud }\end{array}$ & $\begin{array}{l}\text { Número de madres que durante su último embarazo fueron llevadas a un establecimiento } \\
\text { de salud por presentar alguna señal de peligro (fiebre, ardor al orinar, vómitos abundantes, } \\
\text { cefalea, sangrado, hinchazón de pies, manos y cara, bebe no se mueve, pérdida de líquido, } \\
\text { contracciones o dolores fuertes prematuras)/ número de madres encuestadas que presentaron } \\
\text { alguna señal de peligro durante su último embarazo x } 100 \text {. }\end{array}$ \\
\hline 3. Parto institucional & $\begin{array}{l}\text { Número de madres cuyo último parto fue en un establecimiento de salud / número de madres } \\
\text { encuestadas } \times 100 \text {. }\end{array}$ \\
\hline
\end{tabular}

Cuidado durante el puerperio

4. Control posnatal

5. Planificación familiar posparto

Cuidado en recién nacidos

6. Inicio temprano de la lactancia materna

Reconoce señales de peligro

7. en recién nacido y busca ayuda en un establecimiento de salud

Cuidado en niños de 1 a 36 meses

8. Lactancia materna exclusiva

hasta los 6 meses encuestadas $\times 100$

9. Introducción de alimentos sóli-

9. dos/semisólidos

Número de madres que tuvieron control en los siguientes 42 días a su último parto/ número de madres encuestadas $\times 100$.

Número de madres encuestadas que adoptaron un método de planificación familiar en los siguientes 42 días a su último parto dividido / número de madres encuestadas x 100 .

10. Lactancia materna continua

hasta los 2 años

Número de niños menores de 36 meses que fueron amamantados luego de nacer dentro del plazo de una hora/ número de niños encuestados x 100.

Número de niños que fueron llevados al establecimiento de salud durante el primer mes de nacidos por presentar alguna señal de peligro (no puede mamar, ombligo rojo y con pus, pérdida de peso, dificultad respiratoria, diarrea, ictericia)/ número de niños encuestados que presentaron alguna señal de peligro en el primer mes de nacidos $\times 100$

Número de niños menores de 6 meses que recibieron solo lactancia materna el día anterior a la encuesta/ número de niños menores de 6 meses x 100 .

Número de niños de 6 a 8 meses que recibieron alimentos sólidos o semisólidos el día anterior a la encuesta/ número de niños de 6 a 8 meses de edad $x 100$.

11. Suplementación de hierro o

11. multimicronutrientes

Número de niños de 20 a 23 meses que fueron amamantados el día anterior a la encuesta/ número de niños de 20 a 23 meses x 100 .

12. Suplementación con vitamina $A$ Número de niños de 6 a 36 meses que recibieron suplemento de hierro o multimicronutrientes el día anterior a la encuesta/número de niños de 6 a 36 meses x 100.

\section{Reconoce señales de peligro}

13. en niño de 1 a 36 meses y bus-

ca ayuda en un establecimiento de salud

Número de niños de 6 a 36 meses que recibieron suplemento de vitamina $A$ en los 6 meses previos a la encuesta/ número de niños de 6 a 36 meses $\times 100$.

Número de niños de 1 a 36 meses que fueron llevados al establecimiento de salud por presentar alguna señal de peligro (no puede comer o beber, vómitos frecuentes, convulsiones, somnolencia o alguna de las señales de peligro de enfermedad diarreica aguda, infección respiratoria aguda, fiebre, desnutrición o anemia) / número de niños de 1 a 36 meses que presentaron alguna señal de peligro $\times 100$.

14. Cobertura de vacunación en niños de 12 a 23 meses

Número de niños menores de 12 a 23 meses que completaron las vacunas para los primeros 11 meses de edad según calendario de vacunación/ número de niños de 12 a 23 meses x 100 .

\section{Lavado de manos y consumo de agua segura}

15. Lavado de manos con agua y
jabón

16. Consumo de agua segura
Número de padres y/o cuidadores de niños menores de 36 meses que refieren que se lavaron las manos con agua y jabón en los cinco momentos recomendados (antes de preparar los alimentos, antes de comer, antes de dar a comer al niño, después de usar el baño, después de cambiar el pañal al niño)/ número de padres o cuidadores de niños menores de 36 meses $\times 100$.

Número de padres o cuidadores de niños menores de 36 meses que consumen siempre agua segura (clorada o hervida)/ número de padres o cuidadores de niños menores de 36 meses $\mathrm{x} 100$. 
conglomerados multietápicos complejos, que toma en cuenta las probabilidades de muestreo y las ponderaciones muestrales. Los indicadores fueron expresados en porcentajes e intervalos de confianza al 95\% con el ajuste respectivo por diseño muestral, junto a las frecuencias observadas sin ajuste por diseño muestral.

\section{ASPECTOS ÉTICOS}

Por ser el presente estudio de línea de base, parte de una intervención en Salud Pública (estrategia ECPPCES), el protocolo para su realización no fue presentado a un comité de ética. Sin embargo, en cada hogar seleccionado hubo explicación de la encuesta a realizar y se solicitó el consentimiento verbal respectivo. Además, la confidencialidad fue protegida en todo momento separando la información personal de aquella necesaria para el análisis.

\section{RESULTADOS}

Se realizaron encuestas a 540 hogares conformados por 2690 personas (cinco personas/hogar). En 475 hogares (88\%) se encontró, al menos, un niño menor de 3 años, y en 80 hogares $(14,8 \%)$ una mujer gestante. La mayoría de las viviendas de los hogares encuestados tuvieron como material predominante el adobe o tapia $(73,9 \%)$ en paredes, fibra-cemento o calamina $(57,0 \%)$ en techos, y tierra o arena en piso $(80,0 \%)$. Electricidad (78\%) fue la fuente de energía más frecuente de alumbrado y agua entubada $(63,4 \%)$ la principal fuente de agua. En dos tercios de los hogares se cocinaba con leña $(68,0 \%)$, y el pozo ciego y/o silo $(65,1 \%)$ fue el servicio higiénico predominante. Cerca del $90 \%$ de los hogares encuestados fueron clasificados como de extrema pobreza por el método de necesidades básicas insatisfechas (NBI), y el resto como pobres $(10,2 \%)$ (Tabla 2$)$.

Tabla 2. Características de hogares y viviendas encuestadas en áreas con pobreza extrema del Perú, 2012

\begin{tabular}{|c|c|c|c|}
\hline Características & $N=540$ & $(\%)$ & IC $95 \%$ \\
\hline \multicolumn{4}{|l|}{ Material predominante en paredes } \\
\hline Adobe, tapia & 443 & $(73,9)$ & $69,8-77,5$ \\
\hline Madera & 38 & $(11,3)$ & $9,1-13,8$ \\
\hline Ladrillo, cemento & 25 & $(6,2)$ & $4,0-9,4$ \\
\hline Otro (quincha, piedra con barro, estera, etc.) & 34 & $(8,7)$ & $6,1-12,3$ \\
\hline \multicolumn{4}{|l|}{ Material predominante en techo } \\
\hline Eternit, calamina & 365 & $(57,0)$ & $51,8-62,0$ \\
\hline Cemento, tejas & 104 & $(26,7)$ & $22,3-31,7$ \\
\hline Hoja de palmera & 31 & $(10,7)$ & $8,3-13,8$ \\
\hline Otro (paja, estera, caña, quincha, madera, etc.) & 40 & $(5,6)$ & $3,8-8,2$ \\
\hline \multicolumn{4}{|l|}{ Material predominante en piso } \\
\hline Tierra, arena, ripio & 475 & $(80,0)$ & $75,7-83,8$ \\
\hline Cemento & 43 & $(12,8)$ & $9,2-17,5$ \\
\hline Madera & 22 & $(7,1)$ & $5,9-8,5$ \\
\hline \multicolumn{4}{|l|}{ Fuente principal de abastecimiento de agua } \\
\hline Agua entubada & 359 & $(63,4)$ & $58,9-67,6$ \\
\hline Pilón público & 24 & $(2,3)$ & $1,5-3,6$ \\
\hline Otro & 9 & $(1,8)$ & $0,8-4,4$ \\
\hline \multicolumn{4}{|l|}{ Tipo de servicio higiénico } \\
\hline Pozo ciego, silo & 349 & $(65,1)$ & $59,8-70,1$ \\
\hline Letrina & 115 & $(23,2)$ & $18,8-28,3$ \\
\hline Campo abierto & 41 & $(6,4)$ & $4,5-9,1$ \\
\hline Conectado a red pública dentro o fuera de vivienda & 8 & $(0,7)$ & $0,4-1,4$ \\
\hline Otro & 27 & $(4,5)$ & $2,8-7,2$ \\
\hline \multicolumn{4}{|l|}{ Tipo de alumbrado } \\
\hline Electricidad & 414 & $(78,0)$ & $73,8-81,8$ \\
\hline Vela & 86 & $(14,4)$ & $11,1-18,4$ \\
\hline Kerosene, petróleo & 18 & $(6,3)$ & $4,2-9,2$ \\
\hline Otro & 22 & $(1,3)$ & $0,8-2,2$ \\
\hline \multicolumn{4}{|l|}{ Combustible de uso frecuente para cocinar } \\
\hline Leña & 388 & $(68,0)$ & $63,1-72,5$ \\
\hline Bosta (estiércol de animal) & 97 & $(19,4)$ & $16,1-23,1$ \\
\hline Hogares en viviendas con características físicas inadecuadas & 495 & $(86,9)$ & $82,2-90,5$ \\
\hline Hogares en viviendas sin sanitario conectado a alcantarillado & 532 & $(99,3)$ & $98,6-99,6$ \\
\hline Hogares en viviendas con alta dependencia económica & 18 & $(3,0)$ & $1,7-5,2$ \\
\hline Hogares con al menos un niño de 6 a 12 años que no asiste a la escuela & 7 & $(0,8)$ & $0,3-2,1$ \\
\hline Hogares en viviendas con más de 3 personas por habitación para dormir & 234 & $(39,9)$ & $34,9-45,1$ \\
\hline \multicolumn{4}{|l|}{ Hogares según pobreza por método de necesidades básicas insatisfechas (NBI) } \\
\hline Con pobreza (una NBI) & 34 & $(10,2)$ & $7,0-14,7$ \\
\hline Con pobreza extrema (con 2 o más NBI) & 506 & $(89,8)$ & $85,3-93,0$ \\
\hline
\end{tabular}


La Tabla 3 presenta las prácticas de cuidado en el último embarazo de 473 madres de niños menores de 36 meses. La mayoría de las madres $(69,0 \%)$ refirieron haber tenido su primer control prenatal en el primer trimestre de gestación, y haber cumplido más de seis controles prenatales $(65,3 \%)$. La mayoría de mujeres recibieron sulfato ferroso $(83,6 \%)$, sin embargo, su cumplimiento fue variable. Por el contrario, solo una

Tabla 3. Prácticas clave de cuidado durante el embarazo en áreas con pobreza extrema del Perú, 2012

\begin{tabular}{|c|c|c|c|}
\hline Prácticas clave de cuidado & $\mathbf{N}$ & $(\%)$ & IC $95 \%$ \\
\hline \multicolumn{4}{|l|}{ Primer control prenatal $(\mathrm{N}=473)$} \\
\hline Sin CPN & 25 & $(5,5)$ & $3,5-8,6$ \\
\hline Primer trimestre* & 328 & $(69,0)$ & $63,4-74,0$ \\
\hline Segundo trimestre & 99 & $(21,4)$ & $17,0-26,6$ \\
\hline Tercer trimestre & 19 & $(3,8)$ & $2,1-6,8$ \\
\hline NS/NR & 2 & $(0,3)$ & $0,1-2,1$ \\
\hline \multicolumn{4}{|l|}{ Número de CPN durante el embarazo $(\mathrm{N}=473)$} \\
\hline Ninguno & 7 & $(2,3)$ & $1,0-5,1$ \\
\hline 1 a 3 & 22 & $(4,5)$ & $2,6-7,7$ \\
\hline 4 a 6 & 126 & $(24,7)$ & $20,3-29,7$ \\
\hline Más de 6 controles & 300 & $(65,3)$ & $60,0-70,3$ \\
\hline NS/NR & 18 & $(3,2)$ & $1,9-5,4$ \\
\hline \multicolumn{4}{|c|}{ Administración de ácido fólico en $\mathrm{CPN}$ y cumplimiento de tratamiento $(\mathrm{N}=473)$} \\
\hline Sin CPN & 25 & $(5,5)$ & $3,5-8,6$ \\
\hline Administración/cumplimiento en los primeros 3 meses & 49 & $(10,0)$ & $7,1-14,0$ \\
\hline Administración, pero no recuerda periodo de tratamiento & 4 & $(1,4)$ & $0,5-3,7$ \\
\hline No administración & 298 & $(62,7)$ & $57,1-68,0$ \\
\hline NS/NR & 33 & $(8,5)$ & $5,7-12,5$ \\
\hline \multicolumn{4}{|c|}{ Administración de sulfato ferroso en CPN y cumplimiento de tratamiento $(\mathrm{N}=473)$} \\
\hline Sin CPN & 25 & $(5,5)$ & $3,5-8,6$ \\
\hline Administración/cumplimiento en todo el embarazo & 61 & $(11,7)$ & $8,6-15,7$ \\
\hline Administración/cumplimiento mayor parte de embarazo & 159 & $(39,1)$ & $33,7-44,9$ \\
\hline Administración y cumplimiento menor parte del embarazo & 178 & $(32,8)$ & $27,7-38,3$ \\
\hline Administración, pero no recuerda periodo de tratamiento & 2 & $(0,6)$ & $0,1-2,5$ \\
\hline No administración durante CPN & 46 & $(10,2)$ & $7,5-13,8$ \\
\hline NS/NR & 2 & $(0,1)$ & $0,0-0,4$ \\
\hline \multicolumn{4}{|l|}{ Hábito nocivo $(\mathrm{N}=473)$} \\
\hline Tabaquismo, alcohol u otra droga & 32 & $(5,2)$ & $3,5-7,5$ \\
\hline Ningún hábito nocivo & 441 & $(94,8)$ & $92,5-96,5$ \\
\hline \multicolumn{4}{|l|}{ Conducta ante la presencia de señales de peligro $(\mathbf{N}=277)$} \\
\hline Fue a la farmacia & 2 & $(1,1)$ & $0,2-5,6$ \\
\hline Acudió al curandero & 4 & $(0,8)$ & $0,2-2,9$ \\
\hline \multicolumn{4}{|l|}{ Asistencia a sesiones de preparación de parto o psicoprofilaxis $(\mathrm{N}=473)$} \\
\hline No psicoprofilaxis & 352 & $(79,7)$ & $75,2-83,5$ \\
\hline 1-2 sesiones & 76 & $(12,7)$ & $9,6-16,5$ \\
\hline 3-4 sesiones & 35 & $(4,7)$ & $3,1-7,0$ \\
\hline 5 o más sesiones & 4 & $(1,4)$ & $0,4-4,3$ \\
\hline NS/NR & 6 & $(1,6)$ & $0,7-3,8$ \\
\hline \multicolumn{4}{|l|}{ Recibió charla u orientación sobre lactancia materna ( $N=473)$} \\
\hline Sí & 282 & $(56,5)$ & $50,7-62,0$ \\
\hline No & 189 & $(43,1)$ & $37,5-48,8$ \\
\hline NS/NR & 2 & $(0,4)$ & $0,1-1,7$ \\
\hline \multicolumn{4}{|l|}{ Parto institucional $(\mathrm{N}=475)$} \\
\hline Establecimiento de salud (MINSA, ESSALUD, Policía/FFAA, Privado)* & 389 & $(81,1)$ & $76,4-85,1$ \\
\hline Casa materna & 3 & $(1,2)$ & $0,3-4,0$ \\
\hline Domicilio & 83 & $(17,7)$ & $14,0-22,2$ \\
\hline \multicolumn{4}{|l|}{ Planeamiento y cumplimiento del plan de parto $(\mathrm{N}=473)$} \\
\hline Lugar de parto & 292 & $(56,4)$ & $50,8-62,0$ \\
\hline Posición para dar a luz & 257 & $(48,5)$ & $42,9-54,1$ \\
\hline Persona presente durante el parto & 283 & $(54,2)$ & $48,6-59,7$ \\
\hline Participación de pareja o esposo & 251 & $(48,2)$ & $42,6-53,8$ \\
\hline Cosas para llevar en el parto & 303 & $(59,0)$ & $53,4-64,4$ \\
\hline Transporte al momento del parto & 236 & $(46,8)$ & $41,3-52,3$ \\
\hline
\end{tabular}

CPN: control pre natal, NS/NR: no sabe/no responde

*Indicadores de práctica clave de cuidado 
pequeña proporción de mujeres refirieron haber recibido ácido fólico (21,8\%). De las 277 madres que reportaron signos de peligro durante el embarazo, más de la mitad $(56,5 \%)$ refirieron haber acudido a un establecimiento de salud o médico particular. Alrededor del $50 \%$ de madres manifestaron haber elaborado un plan de parto previo al embarazo, y cumplir cada uno de sus ítems al momento del parto. Finalmente, el $81,1 \%$ de las madres refirieron haber dado a luz en un establecimiento de salud.

Del total de madres encuestadas, 17 habían dado a luz recientemente (menos de 30 días posparto). De las 456 madres restantes (Tabla 4), más de la mitad $(58,0 \%)$ refirieron haber tenido el control posnatal dentro de los 42 días, aunque solo un tercio $(31,0 \%)$ habría elegido un método de planificación familiar dentro del mismo periodo. La toma de sulfato ferroso $(38,5 \%)$ y vitamina $A(12,6 \%)$ fue infrecuente. De 155 madres que refirieron signos de peligro durante el puerperio, $52,8 \%$ manifestaron haber acudido a un establecimiento de salud o profesional de salud para atención. Del total de 540 mujeres en edad fértil entrevistadas, solo $12,4 \%$ refirieron haberse lavado las manos con agua y jabón el día de ayer en los cinco momentos recomendados, y $66,4 \%$ reportaron que siempre hierven o agregan cloro al agua antes de tomarla.

Del total de 80 gestantes al momento de la encuesta: 13 estaban en el primer trimestre; 43 en el segundo trimestre, y 24 en el tercer trimestre. Diez $(76,9 \%)$ gestantes en el primer trimestre ya habían iniciado sus controles prenatales, mientras que solo once $(45,8 \%)$ gestantes que se encontraban en el tercer trimestre habían completado seis o más controles prenatales. La administración de ácido fólico y cumplimiento respectivo fue baja en todas las gestantes (15\%), y la administración y cumplimiento de sulfato

Tabla 4. Prácticas clave de cuidado relacionadas al puerperio, lavado de manos y consumo de agua segura en áreas con pobreza extrema del Perú, 2012

\begin{tabular}{|c|c|c|c|}
\hline Prácticas clave de cuidado & $\mathbf{N}$ & $(\%)$ & IC $95 \%$ \\
\hline \multicolumn{4}{|l|}{ Prácticas clave durante puerperio $(\mathrm{N}=456)$} \\
\hline Control posnatal dentro de los 42 días* & 263 & $(58,0)$ & $52,1-63,7$ \\
\hline Elección de método de planificación familiar dentro de los 42 días* & 137 & $(31,0)$ & $25,7-36,9$ \\
\hline Toma de vitamina A por 30 días & 60 & $(12,6)$ & $9,4-16,8$ \\
\hline \multicolumn{4}{|l|}{ Tiempo de toma de sulfato ferroso luego del parto $(\mathrm{N}=456)$} \\
\hline No tomó & 272 & $(60,3)$ & $54,4-66,0$ \\
\hline Un mes & 153 & $(32,2)$ & $27,0-37,9$ \\
\hline Dos meses & 18 & $(4,7)$ & $2,6-8,3$ \\
\hline Más de dos meses & 7 & $(1,6)$ & $0,7-3,9$ \\
\hline NS/NR & 6 & $(1,2)$ & $0,2-2,8$ \\
\hline \multicolumn{4}{|l|}{ Conducta ante la presencia de señales de peligro $(\mathrm{N}=155)$} \\
\hline Se trató en casa & 75 & $(46,6)$ & $36,8-56,7$ \\
\hline Acudió a un establecimiento de salud o profesional de salud* & 78 & $(52,8)$ & $42,8-62,7$ \\
\hline Fue a la farmacia & 1 & $(0,3)$ & $0,0-2,2$ \\
\hline No buscó ayuda & 1 & $(0,2)$ & $0,0-1,7$ \\
\hline \multicolumn{4}{|l|}{ Lavado de manos y consumo de agua segura $(\mathrm{N}=540)$} \\
\hline Antes de preparar los alimentos & 452 & $(86,0)$ & $82,3-89,0$ \\
\hline Antes de comer & 442 & $(83,3)$ & $79,4-86,6$ \\
\hline Antes de dar de comer al niño & 291 & $(52,1)$ & $47,1-57,1$ \\
\hline Después de usar el baño & 296 & $(62,9)$ & $58,4-67,2$ \\
\hline Después de cambiar el pañal al niño & 146 & $(29,8)$ & $25,0-35,1$ \\
\hline En los cinco momentos mencionados arriba* & 44 & $(12,4)$ & $9,0-16,8$ \\
\hline \multicolumn{4}{|l|}{ Práctica en relación al agua para tomar $(\mathrm{N}=540)$} \\
\hline Siempre toma agua hervida o con cloro* & 401 & $(66,3)$ & $61,2-71,0$ \\
\hline Toma agua hervida a veces & 99 & $(23,1)$ & $18,9-28,1$ \\
\hline Toma agua con cloro a veces & 3 & $(1,2)$ & $0,4-3,7$ \\
\hline Toma agua cruda siempre & 36 & $(9,3)$ & $6,5-13,0$ \\
\hline
\end{tabular}

NS/NR: no sabe/no responde

*Indicadores de práctica clave de cuidado 
Tabla 5. Prácticas clave de cuidado del recién nacido en áreas con pobreza extrema del Perú, 2012

\begin{tabular}{|c|c|c|c|}
\hline Prácticas clave en el cuidado del recién nacido & $\mathbf{N}$ & $(\%)$ & IC $95 \%$ \\
\hline \multicolumn{4}{|l|}{ Contacto precoz con pecho materno $(\mathrm{N}=475)$} \\
\hline Menos de una hora & 349 & $(64,1)$ & $58,6-69,3$ \\
\hline Más de una hora o no contacto & 122 & $(35,3)$ & $30,1-40,8$ \\
\hline NS/NR & 4 & $(0,6)$ & $0,2-2,3$ \\
\hline \multicolumn{4}{|l|}{ Inicio de lactancia materna $(\mathrm{N}=475)$} \\
\hline Nunca le dio leche materna & 5 & $(2,0)$ & $0,8-5,3$ \\
\hline Menos de hora luego del parto* & 316 & $(62,8)$ & $57,1-68,1$ \\
\hline De 1 a 11 horas & 125 & $(27,2)$ & $22,5-32,5$ \\
\hline De 12 a 23 horas & 7 & $(1,5)$ & $0,6-3,8$ \\
\hline De 24 a más horas & 18 & $(5,6)$ & $3,2-9,5$ \\
\hline NS/NR & 4 & $(0,9)$ & $0,3-2,8$ \\
\hline \multicolumn{4}{|l|}{ Frecuencia de limpieza de cordón $(\mathrm{N}=475)$} \\
\hline Todos los días & 291 & $(63,2)$ & $57,7-68,4$ \\
\hline Interdiaria & 147 & $(30,5)$ & $25,6-36,0$ \\
\hline Otra frecuencia & 17 & $(2,6)$ & $1,5-4,5$ \\
\hline Nunca lo limpiaron & 18 & $(3,3)$ & $1,9-5,6$ \\
\hline NS/NR & 1 & $(0,4)$ & $0,1-2,8$ \\
\hline \multicolumn{4}{|l|}{ Frecuencia de higiene corporal (N=475) } \\
\hline Nunca & 4 & $(1,2)$ & $0,4-3,6$ \\
\hline Todos los días & 131 & $(26,5)$ & $22,0-31,5$ \\
\hline Interdiaria & 194 & $(42,3)$ & $36,9-47,9$ \\
\hline Dejando dos días & 100 & $(21,8)$ & $17,3-27,1$ \\
\hline Una vez por semana & 31 & $(5,5)$ & $3,7-8,1$ \\
\hline Otra frecuencia & 15 & $(2,7)$ & $1,6-4,8$ \\
\hline \multicolumn{4}{|l|}{ Conducta frente a señales de peligro $(\mathrm{N}=131)$} \\
\hline Fue tratado en casa & 27 & $(19,8)$ & $12,3-30,2$ \\
\hline Fue llevado al establecimiento de salud o profesional de salud* & 107 & $(80,0)$ & $69,3-87,6$ \\
\hline Fue llevado a otro lugar & 1 & $(0,2)$ & $0,0-1,5$ \\
\hline
\end{tabular}

NS/NR: no sabe/ no responde

*Indicadores de práctica clave de cuidado

ferroso el día anterior a la encuesta fue variable de acuerdo al trimestre de gestación: primer trimestre $(23,1 \%)$, segundo trimestre $(58,4 \%)$, y tercer trimestre $(78,3 \%)$.

Cuando se preguntó a las madres de niños menores de 36 meses sobre el cuidado que tuvieron del niño durante su primer mes de nacido (Tabla 5), aproximadamente dos terceras partes $(64,1 \%)$ mencionó haber tenido contacto precoz con su niño, y dado inicio a la lactancia materna (62,8\%). La mayoría de madres (63,2\%) refirieron haber limpiado el cordón todos los días, pero solo el $50 \%$ lo hizo con alcohol puro o yodado. La higiene corporal interdiaria del recién nacido fue la más frecuente $(42,3 \%)$, siendo agua y jabón las sustancias más usadas (88,6\%). De 131 madres que refirieron signos de peligro en sus niños durante el primer mes de nacidos, $80,0 \%$ habrían acudido al establecimiento de salud para la atención respectiva.
La Tabla 6 presenta indicadores de prácticas clave en el cuidado de niños de 1 a 36 meses. El día anterior a la encuesta, 89,6\% niños menores de 6 meses recibieron solo lactancia materna, y $89,1 \%$ niños de 6 a 8 meses ingirieron alimentos sólidos o semisólidos en calidad de alimentación complementaria. Cerca de $60 \%$ niños de 20 a 23 meses fueron a amantados el día previo a la encuesta; y del total de niños entre 6 y 36 meses, 19,6\% habría recibido suplemento de hierro el día previo a la encuesta y $28,5 \%$ vitamina $A$ en los últimos 6 meses. Del total de 170 niños entre 12 y 23 meses, 136 contaban con sus tarjetas de vacunación al momento de la encuesta (80\%). En estos últimos la cobertura completa de vacunación fue de 65,6\%. El porcentaje aumenta a $76,0 \%$ si no se incluye la vacuna con la menor cobertura individual $(79,3 \%)$ : vacuna contra hepatitis-B. Fiebre $(85,9 \%)$, diarrea $(68,5 \%)$, vómitos $(39,2 \%)$ y dificultad respiratoria $(17,4 \%)$ fueron las señales de peligro más referidas para la búsqueda de atención en niños de 1 a 36 meses. 
Tabla 6. Prácticas clave de cuidado del niño de 1 a 36 meses en áreas con pobreza extrema del Perú, 2012

\begin{tabular}{|c|c|c|c|}
\hline Prácticas clave & $\mathbf{N}$ & (\%) & IC $95 \%$ \\
\hline \multicolumn{4}{|l|}{ Indicadores de prácticas clave } \\
\hline Niños menores de 6 meses que recibieron solo lactancia materna el día anterior (lactancia exclusiva) $(\mathrm{N}=64)^{*}$ & 58 & $(89,6)$ & $76,6-95,8$ \\
\hline Niños de 6 a 8 meses de edad que recibieron alimentos sólidos/semisólidos el día anterior $(\mathrm{N}=48)^{*}$ & 43 & $(89,1)$ & $71,5-96,4$ \\
\hline Niños de 20 a 23 meses de edad que fueron amamantados el día anterior $(\mathrm{N}=58)^{*}$ & 33 & $(56,8)$ & $40,5-71,8$ \\
\hline Niños de 6 a 36 meses de edad que recibieron suplemento de hierro o micronutrientes el día anterior $(\mathrm{N}=411)^{*}$ & 79 & $(19,6)$ & $15,0-25,2$ \\
\hline Niños de 6 a 36 meses de edad que recibieron vitamina $A$ en los últimos 6 meses $(N=411)^{*}$ & 122 & $(28,5)$ & $23,2-34,4$ \\
\hline Niños de 12 a 23 meses con cobertura completa de vacunas correspondientes a los primeros 11 meses $(\mathrm{N}=136)^{*}$ & 98 & $(65,6)$ & $54,7-75,1$ \\
\hline \multicolumn{4}{|l|}{ Cobertura estimada de vacunación en niños de 12 a 23 meses ( $N=136)$} \\
\hline BCG & 127 & $(90,6)$ & $81,4-95,5$ \\
\hline Antihepatitis B & 118 & $(79,3)$ & $68,4-87,2$ \\
\hline Polio 1 & 133 & $(96,7)$ & $89,7-99,0$ \\
\hline Polio 2 & 130 & $(93,3)$ & $85,6-97,0$ \\
\hline Polio 3 & 126 & $(89,9)$ & $81,7-94,6$ \\
\hline Penta 1 & 134 & $(98,9)$ & $95,8-99,7$ \\
\hline Penta 2 & 131 & $(95,5)$ & $88,9-98,3$ \\
\hline Penta 3 & 125 & $(90,2)$ & $82,4-94,8$ \\
\hline Rotavirus 1 & 133 & $(97,9)$ & $93,4-99,4$ \\
\hline Rotavirus 2 & 128 & $(94,0)$ & $87,4-97,3$ \\
\hline Neumococo 1 & 132 & $(97,9)$ & $93,6-99,3$ \\
\hline Neumococo 2 & 124 & $(89,6)$ & $81,5-94,5$ \\
\hline \multicolumn{4}{|l|}{ Señales de peligro para búsqueda de atención en niño de 1 a 6 meses $(\mathrm{N}=459)$} \\
\hline Fiebre & 394 & $(85,9)$ & $81,7-89,3$ \\
\hline Diarrea & 310 & $(68,5)$ & $63,0-73,5$ \\
\hline Vómitos & 147 & $(39,2)$ & $33,6-45,1$ \\
\hline Llanto incontrolable & 11 & $(3,7)$ & $1,9-7,1$ \\
\hline No puede beber o lactar & 7 & $(2,4)$ & $1,1-5,4$ \\
\hline Dificultad para respirar & 75 & $(17,4)$ & $13,5-22,0$ \\
\hline Convulsiones & 7 & $(1,8)$ & $0,8-4,0$ \\
\hline Somnolencia & 1 & $(0,1)$ & $0,0-0,6$ \\
\hline Ictericia & 7 & $(1,9)$ & $0,8-4,6$ \\
\hline Desnutrición & 16 & $(5,1)$ & $2,9-8,7$ \\
\hline Anemia & 19 & $(5,3)$ & $3,1-9,0$ \\
\hline
\end{tabular}

*Indicadores de práctica clave de cuidado

\section{DISCUSIÓN}

El presente artículo presenta la evaluación de prácticas clave de cuidado materno-infantil, como parte del estudio de línea de base realizado previo al diseño e implementación de una estrategia de ECPPCES en nueve de los departamentos más pobres del Perú.

La asistencia precoz y periódica a los controles prenatales debe ser universal, por lo que su promoción como práctica clave durante el embarazo debe ser prioritaria en la población. Desafortunadamente, una proporción importante de madres manifestaron haber tenido un control prenatal subóptimo (sin control o con control de inicio tardío e incompleto). El control prenatal representa una oportunidad para incrementar el acceso a un parto en condiciones seguras. Se ha reportado menor incidencia de preeclampsia, infección del tracto urinario, anemia posparto y mortalidad materna cuando los controles prenatales han sido precoces y periódicos. En forma similar, hay menor riesgo de mortalidad perinatal y de nacimientos de niños con bajo peso al nacer ${ }^{(10,11)}$.

Asegurar el suplemento nutricional de la mujer con sulfato ferroso durante todo el periodo embarazo también es una práctica clave por priorizar, considerando el impacto que tiene la anemia en la salud materna e infantil y los bajos niveles de administración y cumplimiento reportados por las madres entrevistadas. El suplemento diario reduce el riego de niños con bajo peso al nacer, incrementa el peso del niño y reduce la mortalidad neonatal (12). No menos importante es el suplemento con acido 
fólico en los primeros 3 meses, poco frecuente en el estudio, que reduce significativamente la incidencia de defectos del tubo neural en el niño.

En la etapa de gestación existen riesgos que ponen en peligro la vida de la mujer y el producto; aproximadamente $15 \%$ de las mujeres embarazadas presentan alguna complicación mortal que requiere de atención obstétrica calificada y en algunos casos intervención obstétrica para que sobrevivan ${ }^{(13)}$. Una complicación no diagnosticada oportunamente puede agravarse y provocar una emergencia obstétrica que incrementa el riesgo de morbimortalidad materno-infantil. Debido a ello, el acudir a un establecimiento de salud inmediato al reconocimiento de señales de peligro durante la gestación también es una práctica clave que debe ser estimulada en la población objetivo ${ }^{(14)}$. Más aun, luego de encontrar que más del $40 \%$ de las madres que han reconocido algún signo de alarma durante el embarazo optaron por no acudir a un establecimiento de salud.

Otra práctica clave que deber ser promovida en la población estudiada es la atención del parto en un establecimiento de salud, dado que un porcentaje aún elevado $(20 \%)$ de madres dieron a luz sin la participación de un profesional de salud. Varios estudios han descrito los beneficios de un parto institucional, resaltando la menor incidencia de complicaciones, morbilidad y mortalidad perinatal y neonatal. Además, se ha descrito la importancia que tienen los controles prenatales en la gestante para incrementar su oportunidad de culminar la gestación con un parto seguro ${ }^{(15,16)}$.

Con relación a la evaluación de las prácticas claves durante el puerperio, tanto el cumplimiento del control posnatal como la elección de método de planificación familiar dentro de los 42 días, evidenciaron frecuencias que requieren ser incrementadas para asegurar el bienestar de la madre y el niño luego del parto ${ }^{(17)}$. La primera práctica pretende identificar signos y síntomas de alarma y disminuir el riesgo de posibles complicaciones durante el puerperio ${ }^{(18)}$. Mientras que la segunda práctica se justifica en el reconocimiento de la importancia del espaciamiento de los embarazos, como una de las medidas para reducir el riesgo de morbilidad y mortalidad materno-infantil (19).

En el cuidado del recién nacido destacan dos prácticas clave que deben ser mejoradas en la población objetivo: el contacto precoz con pecho materno seguido del inicio de la lactancia materna dentro de la primera hora del parto, y la búsqueda inmediata de atención en un establecimiento de salud luego de la identificación de cualquier señal de peligro en el recién nacido. El contacto precoz de la piel de la madre y el recién nacido mejora la efectividad de la primera mamada y reduce el tiempo para lograr una succión efectiva, asimismo, regula o mantiene la temperatura infantil ${ }^{20)}$. En el largo plazo, mejoraría el afecto y apego de la madre e incrementaría la probabilidad de una mayor duración de la lactancia materna ${ }^{(21)}$. Por otro lado, la atención inmediata del recién nacido con signos de peligro en un establecimiento de salud busca la reducción de defunciones en el periodo neonatal, las cuales representan el $40 \%$ del total de defunciones en niños menores de 5 años ${ }^{(22) .}$

El estudio ha evidenciado altas frecuencias de prácticas clave relacionadas a la lactancia materna exclusiva (LME) y la alimentación complementaria en la población objetivo, las cuales deben ser mantenidas y, de ser posible, incrementadas considerando la vasta evidencia sobre sus beneficios. Niños que reciben LME hasta los 3 meses tienen menos incidencia de infecciones gastrointestinales y respiratorias, y cuando tienen infecciones tienden a ser menos graves (23). Otros beneficios para los niños con LME hasta los 6 meses incluyen la reducción del riesgo de muerte súbita del lactante, muerte, obesidad, leucemia, diabetes tipo 1 y 2, y enfermedad celiaca, así como un menor riesgo de enfermedad inflamatoria intestinal en adultos jóvenes ${ }^{(24,25)}$. Por otro lado, después de los 6 meses de edad es más difícil que los niños alimentados exclusivamente con lactancia materna alcancen sus necesidades nutricionales. Por ello, es importante brindarles alimentos complementarios recién preparados que sean ricos en energía y nutrientes, mientras se continúa con la lactancia materna hasta los dos años. El consumo de cantidades suficientes de alimentos complementarios generalmente se traduce en un crecimiento adecuado del niño ${ }^{(20)}$. La lactancia materna después de los 6 meses continúa siendo una fuente de nutrientes esenciales tales como vitamina $A$, calcio y proteína, además, confiere cierta protección contra las enfermedades infecciosas.

Los niños menores de 36 meses requieren una alta ingesta de hierro que muchas veces no se alcanza con la dieta. Las intervenciones para reducir la deficiencia de hierro están entre las intervenciones más efectivas y costo-efectivas en la salud pública ${ }^{(26)}$. La deficiencia de hierro que conlleva a anemia, ha sido asociada a alteraciones en el crecimiento y el desarrollo de la capacidad intelectual del niño ${ }^{(27)}$. Por su parte, el suministro adecuado de suplementos de vitamina A se asocia a la mejoría del sistema inmune y a la reducción de riesgo de infección y muerte ${ }^{(28)}$. Lamentablemente, los resultados muestran frecuencias de esta práctica por debajo del $30 \%$.

Las enfermedades prevenibles mediante vacunación dan cuenta de aproximadamente $10 \%$ de la carga de enfermedad en niños menores de 5 años. Por ello, es 
importante asegurar que reciban el esquema completo de inmunizaciones de manera oportuna, segura y eficaz, a través de servicios accesibles, utilizados y de alta calidad ${ }^{(21)}$. El presente estudio encontró un bajo porcentaje de cobertura completa de vacunación en los niños de 12 a 23 meses. La estrategia ECPPCES debe ayudar a incrementar la cobertura de vacunación en los niños de la población objetivo, sin embargo, sus actividades deben ir de la mano con intervenciones que aseguren la disponibilidad y el acceso a las vacunas en los establecimientos de salud.

El lavado de manos es una medida sencilla y barata que reduce las enfermedades diarreicas ${ }^{(29)}$ y las infecciones respiratorias agudas de manera significativa ${ }^{(30)}$. El consumo de agua segura busca reducir las enfermedades relacionadas al agua contaminada, y la mortalidad que dichas enfermedades producen ${ }^{(21,31)}$. Las intervenciones en la población objetivo deben estar orientadas a incrementar el consumo de agua hervida o clorada y la práctica de lavado de manos con agua y jabón en cada uno de los momentos recomendados: antes de preparar alimentos, antes de comer, antes de dar de comer al niño, después de usar el baño y después de cambiar el pañal al niño ${ }^{(8)}$.

Para la interpretación correcta de los resultados del estudio se deben tener en cuenta las siguientes limitaciones: primero, la muestra es representativa para aquellos indicadores que abarquen el total o mayor parte de los hogares incluidos en el estudio. Cuando se desagregan los indicadores (por grupo de edades en niños, gestantes actuales), las estimaciones pierden representatividad. Segundo, siempre existe la posibilidad de obtener respuestas erróneas de las madres en encuestas de hogares por sesgos de recuerdo en ellas. Esta situación fue posible mitigar la mayoría de veces, recopilando información adicional mediante preguntas diferentes para un mismo periodo de información. Tercero, el artículo no describe las características y valor nutricional de la alimentación recibida por los niños menores de 36 meses y madres gestantes. Por la complejidad del análisis nutricional, los resultados serán presentados en un manuscrito específico. En cuarto lugar, la cobertura de vacunación en niños ha sido estimada considerando la información registrada en los carnés de vacunación que estaban disponibles al momento de la encuesta. No obstante, se ha logrado generar de forma rápida y a un costo relativamente bajo, información útil y de suficiente calidad y exactitud.

En conclusión, dentro del área de intervención del PARSALUD aún existen niveles subóptimos en varias de las prácticas clave para el cuidado de la mujer durante el embarazo (control prenatal y parto institucional) y el puerperio (control posnatal), así como para el cuidado de los recién nacidos (contacto precoz con pecho materno y búsqueda de atención ante señales de peligro) y de los infantes de 1 a 36 meses (suplemento de hierro y vitamina $A$, búsqueda de atención ante señales de peligro, y vacunación). Dichas prácticas requieren ser promovidas y priorizadas por la estrategia ECPPCES para aumentar su frecuencia e intensidad en la población objetivo.

Contribuciones de autoría: JEVH, LSA, WVV y PGH participaron en la concepción y el diseño del estudio, además de la recolección de datos. Todos participaron en el análisis e interpretación de los datos. JEVH, LSA y AR redactaron el artículo y todos los autores revisaron los borradores y la versión final, realizando importantes contribuciones.

Fuentes de financiamiento: la investigación contó con el apoyo del Programa de Apoyo a la Reforma del Sector Salud (PARSALUD II) del Ministerio de Salud, proyecto de inversión pública financiado con recursos del tesoro público y endeudamiento externo proveniente del Banco Interamericano de Desarrollo (BID) y el Banco Mundial (BM).

Conflictos de interés: los autores declaran no tener conflictos de interés

\section{REFERENCIAS BIBLIOGRÁFICAS}

1. Instituto Nacional de Estadística e Informática. Informe Técnico: Evolución de la Pobreza Monetaria 2007-2012 [Internet]. Lima: INEI; 2013 [citado el 20 de agosto del 2013]. Disponible en: http://www. inei.gob.pe/media/cifras_de_pobreza/pobreza_informetecnico2013_1.pdf

2. Perú, Ministerio de Salud. La mortalidad materna en el Perú 2002-2011. Lima: Dirección General de Epidemiología; 2013.

3. Instituto Nacional de Estadística e Informática. Encuesta Demográfica y de
Salud Familiar 2012 [Internet]. Lima: INEI; 2013 [citado el 20 de agosto del 2013]. Disponible en: http://desa.inei. gob.pe/endes/images/ppr2012_1.pdf

4. Instituto Nacional de Estadística e Informática. Indicadores de Resultados de los Programas Estratégicos, 2000 - 2012 [Internet]. Lima: INEI; 2013 [citado el 20 de agosto del 2013]. Disponible en: http://www.midis. gob.pe/dgsye/datal/files/enic/ eje1/documento-tecnico/ENDESPPR2012.pdf
5. Perú, Ministerio de Salud. Estudio de Factibilidad del Programa de Apoyo a la Reforma del Sector Salud [Internet]. Lima: PARSALUD; 2008 [citado el 20 de agosto del 2013]. Disponible en: http://www. parsalud.gob.pe/index.php/factibilidaddel-programa?download=508: resumenejecutivo

6. Instituto Nacional de Estadística e Informática. Sistema de Difusión de los Censo Nacional. Censo de Población y Viviendas 2007 [Internet]. Lima: INEI; 2013 [citado el 20 de agosto del 
2013]. Disponible en: http://ineidw. inei.gob.pe/ineidw/\#

7. La mortalidad materna en el Perú. Bol Epidemiol. 2012;21(52):892-6.

8. Perú, Ministerio de Salud. Documento Técnico: Promoción de prácticas y entornos saludables para el cuidado infantil [Internet]. Lima: Dirección General de Promoción de la Salud; 2011 [citado el 20 de agosto del 2013]. Disponible en: ftp://ftp2.minsa.gob.pe/descargas/ dgps/compendio/pdf/RM52_-201_ SA_Cuidado_Infantil.pdf

9. Feres JC, Mancero X. El Método de las Necesidades Básicas Insatisfechas (NBI) y sus Aplicaciones en América Latina. Serie estudios estadísticos y prospectivos 7. Santiago de Chile: CEPAL; 2001.

10. Carroli G, Villar J, Piaggio G, KhanNeelofur D, Gülmezoglu M, Mugford $\mathrm{M}$, et al. WHO systematic review of randomised controlled trials of routine antenatal care. Lancet. 2001 May 19;357(9268):1565-70.

11. Raatikainen K,Heiskanen N, Heinonen S. Under-attending free antenatal care is associated with adverse pregnancy outcomes. BMC Public Health. 2007 Sep 27;7:268.

12. Zeng L, Dibley MJ, Cheng Y, Dang S, Chang S, Kong L, et al. Impact of micronutrient supplementation during pregnancy on birth weight, duration of gestation, and perinatal mortality in rural western China: double blind cluster randomised controlled trial. BMJ. 2008 Nov 7;337:a2001. doi: 10.1136/ bmj.a2001.

13. Organización Mundial de la Salud. Manejo de las complicaciones del embarazo y el parto: Guía para obstetrices y médicos. Ginebra: OMS; 2000.

14. Organización Panamericana de la Salud. AIEPI Neonatal: Intervenciones Basadas en Evidencia en el contexto del continuo materno-recién nacido-niño menor de 2 meses. Washington: OPS; 2009.

15. Say L, Raine R. A systematic review of inequalities in the use of maternal health care in developing countries: examining the scale of the problem and the importance of context.
Bull World Health Organ. 2007 Oct; 85(10):812-9.

16. Harvey SA, Blandón YC, McCawBinns A, Sandino I, Urbina L, Rodríguez $\mathrm{C}$, et al. Are skilled birth attendants really skilled? A measurement method, some disturbing results and a potential way forward. Bull World Health Organ. 2007 Oct;85(10):783-90.

17. World Health Organization, United Nations Children's Fund. Home visits for the newborn child: a strategy to improve survival. Geneva: WHO and UNICEF; 2009.

18. Bashour HN, Kharouf $\mathrm{MH}, \mathrm{Ab}$ dulsalam AA, El Asmar K, Tabbaa MA, Cheikha SA. Effect of postnatal home visits on maternal/infant outcomes in Syria: a randomized controlled trial. Public Health Nurs. 2008 Mar-Apr;25(2):115-25. doi: 10.1111/j.1525-1446.2008.00688.x.

19. Grisaru-Granovsky S, Gordon ES, Haklai Z, Samueloff A, Schimmel MM. Effect of interpregnancy interval on adverse perinatal outcomes--a national study. Contraception. 2009 Dec;80(6):512-8. doi: 10.1016/j.contraception.2009.06.006.

20. Moore ER, Anderson GC, Bergman N, Dowswell T. Early skin-to-skin contact for mothers and their healthy newborn infants. Cochrane Database Syst Rev. 2012 May 16;5:CD003519. doi: 10.1002/14651858.CD003519.pub3.

21. Hill Z, Kirkwood B, Edmond K. Prácticas familiares y comunitarias que promueven la supervivencia, el crecimiento y el desarrollo del niño: Evidencia de las intervenciones. Washington: OPS; 2005.

22. Lawn JE, Cousens S, Zupan J; Lancet Neonatal Survival Steering Team. 4 million neonatal deaths: When? Where? Why? Lancet. 2005 Mar 5-11;365(9462):891-900.

23. Sargent J, Dalton M, Schwartz L. Health benefits of breastfeeding promotion. JAMA 2001; 285:2446-7.

24. Arifeen S, Black RE, Antelman G, Baqui A, Caulfield L, Becker S. Exclusive breastfeeding reduces acute respiratory infection and diarrhea deaths among infants in Dhaka slums. Pediatrics. 2001 Oct;108(4):E67.

25. Effect of breastfeeding on infant and child mortality due to infectious diseases in less developed countries: a pooled analysis. WHO Collaborative Study Team on the Role of Breastfeeding on the Prevention of Infant Mortality. Lancet. 2000 Feb 5;355(9202):451-5.

26. Sanabria H, Tarqui C. Fundamentos para la fortificación de la harina de trigo con micronutrientes en el Perú. An Fac Med. 2007;68(2):185-92.

27. De-Regil LM, Suchdev PS, Vist GE, Walleser S, Peña-Rosas JP. Home fortification of foods with multiple micronutrient powders for health and nutrition in children under two years of age (Review). Evid Based Child Health. 2013 Jan;8(1):112-201. doi: 10.1002/ ebch.1895.

28. Beaton GH, Martorell R, Aronson KJ, Edmonston B, McCabe G, Ross AC, et al. Effectiveness of vitamin A supplementation in the control of young child morbidity and mortality in developing countries. ACC/SCN State of the Art Series, Nutrition Policy Discussion Paper No. 13. Geneva: ACC/Subcommittee on Nutrition; 1993.

29. Ejemot RI, Ehiri JE, Meremikwu MM, Critchley JA. Hand washing for preventing diarrhoea. Cochrane Database Syst Rev. 2008 Jan 23;(1):CD004265. doi: 10.1002/14651858.CD004265. pub2.

30. Luby SP, Halder AK, Huda TM, Unicomb L, Johnston RB. Using child health outcomes to identify effective measures of handwashing. Am J Trop Med Hyg. 2011 Nov;85(5):882-92. doi: 10.4269/ajtmh.2011.11-0142.

31. Caruso B, Stephenson R, Leon JS. Maternal behavior and experience, care access, and agency as determinants of child diarrhea in Bolivia. Rev Panam Salud Publica. 2010;28(6):429-39.

Correspondencia: José Velásquez Hurtado Dirección: Av. Paso de los Andes 681. Pueblo Libre, Lima 21 - Perú.

Teléfono: (511)998674127

Correo electrónico:jose.velasquez@upch.pe 